\title{
Erratum: Calculation of the pion-photon transition form factor using dispersion relations and renormalization-group summation [Phys. Rev. D 98, 096017 (2018)]
}

\author{
César Ayala, S. V. Mikhailov®, and N. G. Stefanis \\ (Received 17 February 2020; accepted 21 February 2020; published 5 March 2020)
}

DOI: $10.1103 /$ PhysRevD.101.059901

The graphics shown in the right panels of Figs. 1 and 2 of the journal version are the result of an erroneous computer code. The corresponding corrected curves are displayed here in Fig. 1.

Analogously, also the predictions shown in Fig. 3 in the journal are wrong. The correct contribution to the transition form factor (TFF), comprising the twist-two and twist-four terms, is shown as a dashed line in Fig. 2 below.
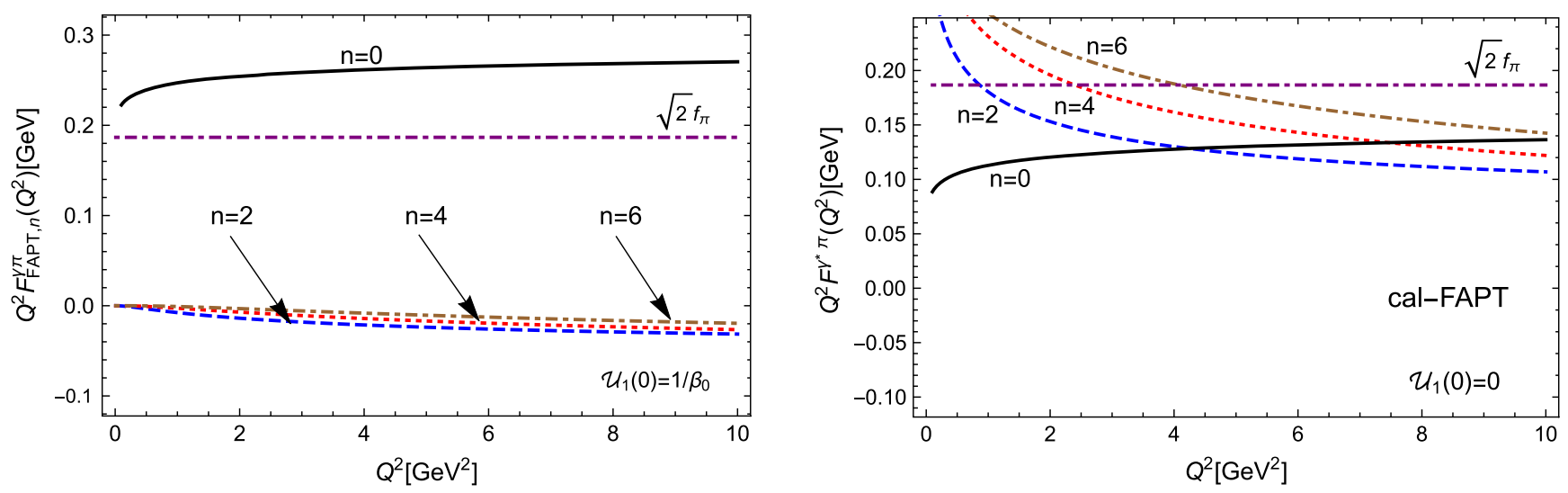

FIG. 1. Corrected graphical results of the right panels of Figs. 1 (left) and 2 (right) of the journal version. The curves in the left panel were calculated with the modified FAPT scheme, $Q^{2} F_{\text {FAPT }, n}^{\gamma \pi}\left(Q^{2}\right)$, according to Eq. (22) for $\mathfrak{A}_{1}^{(1)}(0)=1 / \beta_{0}$ from (20b), and with $m^{2}=4 m_{\pi}^{2} \approx 0.08 \mathrm{GeV}^{2}$ from Eq. (20a) for the higher harmonics. Those in the right panel follow from Eq. (22) in cal-FAPT with $\mathfrak{A}_{\nu}(0)=\mathcal{A}_{\nu}(0)=0$ according to Eq. (20b).

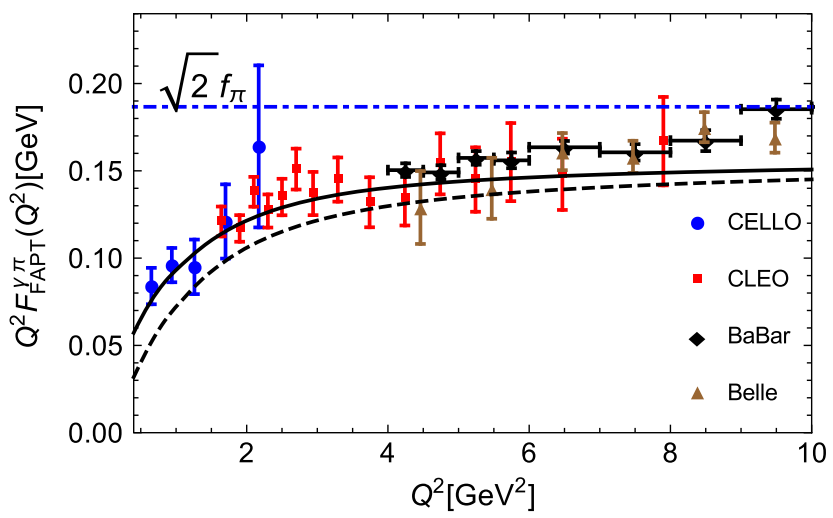

FIG. 2. Theoretical prediction from Eq. (38) for the scaled $\gamma^{*} \gamma \pi^{0}$ transition form factor $Q^{2} F_{\text {FAPT }}^{\gamma \pi}\left(Q^{2}\right)$ using the BMS DA [1]-dashed line-shown in comparison with various experimental data up to $10 \mathrm{GeV}^{2}$ with labels as indicated in the figure. The solid line shows the analogous theoretical prediction obtained by including into the TFF the twist-six contribution.

Published by the American Physical Society under the terms of the Creative Commons Attribution 4.0 International license. Further distribution of this work must maintain attribution to the author(s) and the published articles title, journal citation, and DOI. 
As one sees, fractional analytic perturbation theory (FAPT) resummation of the radiative corrections induces a sizeable suppression of the transition form factor. To get agreement with the experimental data, it is mandatory to include into expression (36) the twist-six contribution. This is done by taking into account the spectral density $\bar{\rho}\left(Q^{2}, x\right)$ for the initial light-cone sum rules in Eq. (28), the corresponding contribution $\bar{\rho}_{\mathrm{tw}-6}[2,3]$, given by

$$
\bar{\rho}_{\mathrm{tw}-6}\left(Q^{2}, x\right)=8 \pi \frac{C_{F}}{N_{c}} \frac{\alpha_{s}\langle\bar{q} q\rangle^{2}}{f_{\pi}^{2}} \frac{x}{Q^{4}}\left[-\left(\frac{1}{1-x}\right)_{+}+(2 \delta(\bar{x})-4 x)+(3 x+2 x \log x+2 x \log \bar{x})\right],
$$

where $\alpha_{s}=0.494$ and $\langle\bar{q} q\rangle^{2}=(0.240 \pm 0.01)^{6} \mathrm{GeV}^{6}$ [2]. The resulting TFF is shown in the figure in terms of a solid line. Thus, the conclusion drawn in the journal version that one gets agreement with the data by taking into account only the twist-two and twist-four terms has to be corrected accordingly.

The deviations of the results given in Figs. 4 and 5 of the journal version differ from their corrected counterparts by less than about 5 percent and are therefore not shown here.

\section{ACKNOWLEDGMENTS}

We would like to thank Alex Pimikov for help with the numerical computations.

[1] A. P. Bakulev, S. V. Mikhailov, and N. G. Stefanis, Phys. Lett. B 508, 279 (2001); [590, 309(E) (2004)].

[2] S. S. Agaev, V. M. Braun, N. Offen, and F. A. Porkert, Phys. Rev. D 83, 054020 (2011).

[3] S. V. Mikhailov, A. V. Pimikov, and N. G. Stefanis, Phys. Rev. D 93, 114018 (2016). 